\title{
Resveratrol Has Antiinflammatory and Antifibrotic Effects in the Peptidoglycan-Polysaccharide Rat Model of Crohn's Disease
}

\author{
Kinan Rahal, MD, ${ }^{*}$ Phyllissa Schmiedlin-Ren, MD, ${ }^{*}$ Jeremy Adler, MD, MSC, ${ }^{+}$ \\ Muhammad Dhanani, $B A,{ }^{*}$ Victoria Sultani, $B A,{ }^{*}$ Ahren C. Rittershaus, $M D_{,}^{*}$ Laura Reingold,* \\ Ji Zhu, PhD, Barbara J. McKenna, MD, ${ }^{\ddagger}$ Gregory M. Christman, MD, and Ellen M. Zimmermann, MD*
}

\begin{abstract}
Background: Resveratrol has antiinflammatory and antifibrotic effects. Resveratrol decreases proliferation and collagen synthesis by intestinal smooth muscle cells. We hypothesized that resveratrol would decrease inflammation and fibrosis in an animal model of Crohn's disease.

Methods: Peptidoglycan-polysaccharide (PG-PS) or human serum albumin (HSA) was injected into the bowel wall of Lewis rats at laparotomy. Resveratrol or vehicle was administered daily by gavage 1-27 days postinjection. On day 28, gross abdominal and histologic findings were scored. Cecal collagen content was measured by colorimetric analysis of digital images of trichrome-stained sections. Cecal levels of procollagen, cytokine, and growth factor mRNAs were determined.

Results: PG-PS-injected rats (vehicle-treated) developed more fibrosis than HSA-injected rats by all measurements: gross abdominal score $(P<$ $0.001)$, cecal collagen content $(P=0.04)$, and procollagen I and III mRNAs $(P \leq 0.0007)$. PG-PS-injected rats treated with $40 \mathrm{mg} / \mathrm{kg}$ resveratrol showed a trend toward decreased gross abdominal score, inflammatory cytokine mRNAs, and procollagen mRNAs. PG-PS-injected rats treated with $100 \mathrm{mg} / \mathrm{kg}$ resveratrol had lower inflammatory cytokine mRNAs (IL-1 $\beta[3.50 \pm 1.08$ vs. $10.79 \pm 1.88, P=0.005]$, IL-6 [17.11 \pm 9.22 vs. $45.64 \pm 8.83, P=0.03]$, tumor necrosis factor alpha (TNF- $\alpha)[0.80 \pm 0.14$ vs. $1.89 \pm 0.22, P=0.002])$, transforming growth factor beta 1 (TGF- $\beta 1)$ mRNA $(2.24 \pm 0.37$ vs. $4.06 \pm 0.58, P=0.01)$, and histologic fibrosis score $(6.4 \pm 1.1$ vs. $9.8 \pm 1.0 ; P=0.035)$ than those treated with vehicle. There were trends toward decreased gross abdominal score and decreased cecal collagen content. Procollagen I, procollagen III, and IGF-I mRNAs also trended downward.
\end{abstract}

Conclusions: Resveratrol decreases inflammatory cytokines and TGF- $\beta 1$ in the PG-PS model of Crohn's disease and demonstrates a promising trend in decreasing tissue fibrosis. These findings may have therapeutic applications in inflammatory bowel disease.

(Inflamm Bowel Dis 2012;18:613-623)

Key Words: inflammatory bowel disease, fibrosis, stricture, inflammation

Received for publication June 28, 2011; Accepted July 5, 2011.

From the *Department of Internal Medicine, Division of Gastroenterology, University of Michigan, Ann Arbor, Michigan, ${ }^{\dagger}$ Department of Pediatrics, Division of Gastroenterology, University of Michigan, Ann Arbor, Michigan, Department of Pathology, University of Michigan, Ann Arbor, Michigan, ${ }^{\S}$ Department of Statistics, University of Michigan, Ann Arbor, Michigan, "Department of Obstetrics and Gynecology, University of Michigan, Ann Arbor, Michigan.

Supported by a grant from the National Institutes of Health (1RO1DK073992 to E.M.Z.).

Current address for Kinan Rahal: Department of Internal Medicine, Division of Gastroenterology, Texas A\&M Health Science Center College of Medicine, Temple, Texas.

Current address for Victoria Sultani: Ohio State University College of Medicine, Columbus, Ohio.

Reprints: Ellen M. Zimmermann, MD, University of Michigan, Department of Internal Medicine, Division of Gastroenterology, Room 6520 MSRB I/SPC 5682, 1150 W. Medical Center Dr., Ann Arbor, MI 48109 (e-mail: ezimmer@umich. edu).

Copyright $(\subset) 2011$ Crohn's \& Colitis Foundation of America, Inc. DOI 10.1002/ibd.21843

Published online 29 August 2011 in Wiley Online Library (wileyonlinelibrary.com).
$\mathrm{T}$ he natural history of Crohn's disease (CD) is characterized by segmental, transmural bowel wall inflammation that waxes and wanes over years and often progresses to fibrosis, critical luminal narrowing, and obstruction. There are currently no effective medical therapies for fibrotic strictures in $\mathrm{CD}$, leaving surgery as the only treatment for symptomatic strictures.

Resveratrol (3,4',5-trihydroxy-trans-stilbene) is a phytoalexin present in the skin of red grapes and in peanuts and blueberries. ${ }^{1}$ Previous reports have demonstrated that resveratrol has antioxidant, ${ }^{2-4}$ antiaging, ${ }^{4,5}$ and cancer chemopreventive properties. ${ }^{1,2,4,6-9}$ Relevant to $\mathrm{CD}$, resveratrol also has antiinflammatory and antifibrotic effects. ${ }^{10-18}$ Multiple in vivo animal studies have demonstrated the antiinflammatory effects of resveratrol in several organ systems, ${ }^{10,11}$ including animal models of gastrointestinal tract inflammation. ${ }^{12-18}$

Resveratrol has been shown to have an antifibrotic effect in animal models of cirrhosis, ${ }^{19-21}$ renal fibrosis, ${ }^{22}$ 
cardiac fibrosis, ${ }^{23}$ and vasculopathy. ${ }^{24,25}$ With regard to the gastrointestinal tract, resveratrol protected against stricture formation following experimental caustic esophageal burns. ${ }^{18}$ To our knowledge, the antifibrotic effects of resveratrol have not been studied in inflammatory bowel disease (IBD) or models thereof.

We previously showed that resveratrol decreases proliferation and collagen I synthesis by rat intestinal smooth muscle cells in vitro. ${ }^{26}$ The aim of this study was to determine if resveratrol has antiinflammatory and antifibrotic effects in the peptidoglycan-polysaccharide (PG-PS) rat model of $\mathrm{CD}$, which exhibits chronic intestinal inflammation and prominent fibrosis. ${ }^{27,28}$

\section{MATERIALS AND METHODS}

\section{Animals}

These studies were approved by the University Committee on Use and Care of Animals at the University of Michigan. Specific pathogen-free (SPF) female Lewis rats, $8-10$ weeks of age and weighing $\approx 150-165 \mathrm{~g}$, were obtained from Harlan Laboratories (Indianapolis, IN). The animals were housed under SPF and temperature-controlled conditions, with light/dark cycles of 12/12 hours and free access to water and standard rodent chow.

\section{PG-PS Enterocolitis Model}

As previously described, ${ }^{27,28}$ anesthetized rats underwent laparotomy. With aseptic technique, intramural injections of PG-PS (15 $\mu \mathrm{g}$ rhamnose/g body weight; PG-PS 10S from Lee Laboratories / Becton Dickinson, Bedford, MA) were administered at seven sites along the surgically exposed intestine (in ileal Peyer's patches, terminal ileum, and cecum) using $33 \mathrm{G}$ needles. Control rats were injected with human serum albumin (HSA; $45 \mu \mathrm{g} / \mathrm{g}$ body weight; sterile solution in normal saline) in the same manner. After the operation, the animals were closely monitored, weighed three times per week, and allowed free access to rodent chow and water. In this model, PG-PS induces transient acute inflammation, followed by a quiescent phase, then a spontaneously reactivating chronic granulomatous phase which begins by day 14 and is associated with chronic intestinal inflammation and prominent fibrosis. ${ }^{27-29}$

\section{Study Design}

In each experiment there were four groups of 4-6 rats/ group: HSA-injected, given vehicle; HSA-injected, given resveratrol; PG-PS-injected, given vehicle; PG-PS-injected, treated with resveratrol.

\section{Resveratrol Administration}

Resveratrol treatment by daily gavage was begun on day 1 postinjection. Three separate experiments were conducted: $20 \mathrm{mg} / \mathrm{kg} / \mathrm{d}, 40 \mathrm{mg} / \mathrm{kg} / \mathrm{d}$, and $100 \mathrm{mg} / \mathrm{kg} / \mathrm{d}$ resveratrol dosing. Resveratrol (Cat. no. R5010, >99\% purity by GC; Sigma Chemical, St. Louis, MO) was dissolved in absolute alcohol at a concentration of $50 \mathrm{mg} / \mathrm{mL}$. This stock solution was stored at $-20^{\circ} \mathrm{C}$ in the dark. Immediately prior to use the required volume of resveratrol stock solution was mixed with sufficient $1 \%$ carboxymethyl cellulose (CMC) in water to give a gavage volume of $0.4 \mathrm{~mL}$ (for $20 \mathrm{mg} / \mathrm{kg}$ or $40 \mathrm{mg} / \mathrm{kg}$ dose) or $1.0 \mathrm{~mL}$ (for $100 \mathrm{mg} / \mathrm{kg}$ dose). The control rats received the same volume of alcohol in 1\% CMC.

\section{Gross Abdominal Score}

On day 28 postinjection the animals were euthanized. The abdomen was opened and gross findings were scored in a blinded manner. The "gross abdominal score" was calculated as the sum of the values (on 0-4 scales) assigned to: 1) number of cecal nodules; 2) extent of cecal wall thickening based on the degree of opacity of the bowel wall and the perceived thickness on palpation; 3) severity of thickening and contraction of the cecal and terminal ileal mesentery; 4) severity of adhesions; and 5) number of liver nodules. The maximum possible score representing the most severe lesions is $20{ }^{28}$

\section{Histologic Assessment}

At the time of dissection a $1 \times 2 \mathrm{~cm}$ portion of the wall of the cecum was resected. One half of this piece of tissue was shaped into a Swiss roll and placed in $10 \%$ neutral buffered formalin; the remaining half was frozen for RNA analysis (see below). A $0.5-\mathrm{cm}$ length of the cecal tip was collected and placed in formalin. After 24 hours the tissue was removed from formalin, embedded in paraffin, and sectioned. From each tissue sample, one section was stained with hematoxylin and eosin (H\&E) and another with a modification of Masson's original trichrome stain. This trichrome system stains collagen blue, nuclei purple-brown, and cytoplasm pink. All slides were scored by a gastrointestinal pathologist (A.C.R.) in a blinded manner for inflammation and fibrosis based on the scoring system shown in Table 1. The inflammation score for a given tissue section is the sum of the scores given to the four regions or features assessed for inflammation (four columns under "Inflammation"). The fibrosis score for a given tissue section is the sum of the scores given to the four regions assessed for fibrosis (four columns under "Fibrosis").

\section{Colorimetric Analysis of Digital Images of Trichrome-stained Sections for Cecal Wall Collagen Content}

Colorimetric analysis was done as previously described on the trichrome-stained sections of cecum. ${ }^{29}$ Briefly, digital images of the stained tissue sections were captured using a Polaroid SprintScan 35 Plus (model CS-3600) and recorded in tagged image file (TIF) format. Image analysis was performed on Mac OS 10.5.6. The image white background was removed using Adobe Photoshop CS3 v. 10.0.1 (San Jose, CA). Color segmentation analysis was then performed with MatLab 
TABLE 1. Histologic Scoring: Inflammation and Fibrosis

\begin{tabular}{|c|c|c|c|c|c|c|c|c|}
\hline \multirow[b]{2}{*}{ Score } & \multicolumn{4}{|c|}{ Inflammation } & \multicolumn{4}{|c|}{ Fibrosis } \\
\hline & $\begin{array}{c}\text { Mucosal } \\
\text { (Crypt } \\
\text { Abscesses) }\end{array}$ & Submucosal & Serosal & $\begin{array}{l}\text { Granulomas } \\
\text { (no.) }\end{array}$ & Submucosal & Serosal & $\begin{array}{l}\text { Muscularis } \\
\text { Mucosae }\end{array}$ & $\begin{array}{c}\text { Muscularis } \\
\text { Externa }\end{array}$ \\
\hline 0 & None & None & None & 0 & None & None & None & None \\
\hline 1 & Focal & Focal & Focal & $1-2$ & Focal & Focal & Focal & Focal \\
\hline 2 & Multifocal & Patchy & Patchy & $3-4$ & Patchy & Patchy & Patchy & Patchy \\
\hline 3 & $\begin{array}{c}\text { Confluent } \\
\text { patchy }\end{array}$ & Diffuse & Diffuse & $5-9$ & Diffuse & Diffuse & Diffuse & Diffuse \\
\hline 4 & Diffuse & $\begin{array}{l}\text { Diffuse } \\
\text { severe }\end{array}$ & $\begin{array}{l}\text { Diffuse } \\
\text { severe }\end{array}$ & $\begin{array}{l}\text { Many/ } \\
\text { necrotizing }\end{array}$ & $\begin{array}{l}\text { Diffuse } \\
\text { severe }\end{array}$ & $\begin{array}{l}\text { Diffuse } \\
\text { severe }\end{array}$ & $\begin{array}{l}\text { Diffuse } \\
\text { severe }\end{array}$ & $\begin{array}{l}\text { Diffuse } \\
\text { severe }\end{array}$ \\
\hline
\end{tabular}

software (R2009a; MathWorks, Natick, MA). Colors were then converted into HSV (hue, saturation, value) color space. $^{30,31}$ Each pixel was categorized by color classification to identify its likely tissue type. Collagen area was defined as the distinct blue color region and was distinguished from muscle, blood, and inflammatory cells. The total length of each tissue section was measured. In cases of curved tissue, multisegment measurements were performed along the axis of the muscularis propria, without regard to plicae. All linear measurements were performed by J.A. on the digital images, using Osirix software. The collagen area of each tissue section was then normalized to tissue section size by dividing the number of pixels in the blue collagen area by the length of the section. Prior studies demonstrated an excellent correlation between the results of colorimetric analysis of digital images of trichrome-stained sections for cecal wall collagen content and measurement of collagen type I content by western immunoblot. $^{29}$

\section{Messenger RNA Analysis}

One half of the $1 \times 2 \mathrm{~cm}$ portion of PG-PS- or HSAinjected cecum, harvested at the time of dissection as described above, was snap-frozen in liquid nitrogen and placed at $-80^{\circ} \mathrm{C}$ for later RNA extraction. RNA was isolated from pulverized frozen cecal tissue using the RNeasy Midi Kit (Qiagen, Valencia, CA) according to the manufacturer's instructions. The RNA concentration was calculated from the absorbance at $260 \mathrm{~nm}$. A portion of the RNA was reversetranscribed into cDNA using the High Capacity cDNA Reverse Trancription Kit (Applied Biosystems, Foster City, CA) according to the manufacturer's instructions. cDNAs were used in quantitative real-time polymerase chain reactions (PCR) for procollagen types I and III (using primers and probes previously designed by our laboratory to span an intron $^{26}$ ) as well as insulin-like growth factor I (IGF-I), transforming growth factor beta 1 (TGF- $\beta 1$ ), tumor necrosis factor alpha (TNF- $\alpha$ ), interleukin-1 beta (IL-1 $\beta$ ), and interleukin-6 (IL-6) (using commercially available TaqMan Gene Expression Assays from Applied Biosystems). Results were normal- ized to glyceraldehyde 3-phosphate dehydrogenase (GAPDH) mRNA levels (measured using commercially available TaqMan Gene Expression Assay); then fold change was calculated as fold of the mean vehicle-treated HSA-injected control value. All reactions were done in triplicate wells.

\section{Statistical Analysis}

Experimental results were assessed for significance using one-tailed unpaired $t$-tests, and dose-response relationships were determined by linear regression. $P<0.05$ was considered significant.

\section{Ethical Considerations}

Animal use complied with the Guide for the Care and Use of Laboratory Animals (Institute of Laboratory Animal Resources, Commission on Life Sciences, National Research Council; National Academies Press, Washington, DC, 2010; ISBN-10: 0-309-15400-6).

\section{RESULTS}

\section{PG-PS-injected vs. HSA-injected Rats}

When PG-PS-injected (vehicle-treated) or HSAinjected (vehicle-treated) rats from all three experiments were combined, the gross abdominal score was greater in PG-PS-injected than in HSA-injected animals (Fig. 1A; $11.93 \pm 1.42$ vs. $0.20 \pm 0.11$, respectively; $P<$ $0.00000001 ; n=15$ /group). Histologic scores for both inflammation and fibrosis on $\mathrm{H} \& \mathrm{E}$ and trichrome-stained sections were greater in PG-PS-injected than in HSAinjected animals in the two experiments for which this was performed (40 and $100 \mathrm{mg} / \mathrm{kg} / \mathrm{d}$ experiments; Fig. 1B; inflammation score: open bars, $11.00 \pm 0.82$ vs. $0 \pm 0$, respectively, $P<0.00000001$; fibrosis score: filled bars, $8.11 \pm 0.68$ vs. $0.11 \pm 0.11$, respectively, $P<$ $0.00000001 ; n=9$ /group). The collagen content of cecal tissue as measured by colorimetric analysis of digital images of trichrome-stained sections and expressed relative 

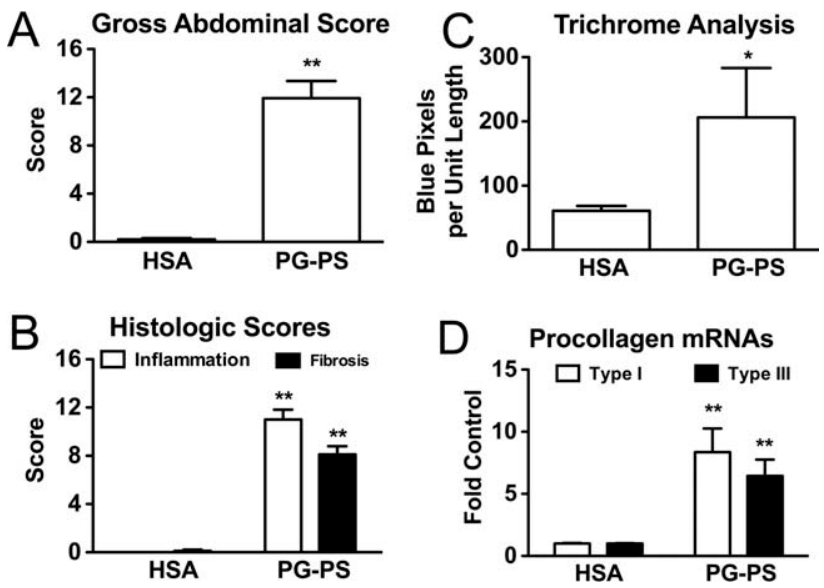

FIGURE 1. By all measurements used, vehicle-treated PG-PSinjected rats developed more fibrosis at day 28 postinjection than did vehicle-treated HSA-injected rats. All results shown are means; error bars reflect SEM. (A) PG-PS-injected animals had greater gross abdominal scores $(P<0.001)$, which are the sums of the scores for adhesions, cecal wall thickening, mesenteric thickening and contraction, cecal nodules, and liver nodules, each graded on a scale of $0-4$, with a maximum possible score of 20 ( $n=15 /$ group; combined data from all three experiments). (B) PG-PS-injected animals had greater histologic inflammation and fibrosis scores when H\&E and trichrome-stained sections of cecum were examined in a blinded manner and scored for the amounts of inflammation and fibrosis as described in Materials and Methods (inflammation score, open bars; fibrosis score, filled bars; maximum possible score for each is 16; $P<0.00000001$ for both; $n=9$ /group; combined data from the 40 and $100 \mathrm{mg} / \mathrm{kg} / \mathrm{d}$ experiments). (C) The collagen content of cecal tissue, as analyzed by colorimetric analysis of digital images of trichrome-stained sections, was also greater in PG-PSinjected than in HSA-injected animals $(P<0.02)$. Collagen is stained blue in the trichrome system used; thus, blue pixels reflect collagen. Variation in the sizes of the pieces of tissue analyzed was controlled for by normalizing to tissue length. ( $n=9 / \mathrm{group}$; combined data from the 40 and $100 \mathrm{mg} / \mathrm{kg} / \mathrm{d}$ experiments) (D) Cecal tissue of PG-PS-injected rats had increased procollagen type I (open bars; $P<0.001$ ) and procollagen type III (filled bars; $P<$ $0.001)$ mRNA expression as measured by quantitative real-time PCR; the results were normalized to GAPDH and expressed as fold the mean HSA-injected control value ( $n=9$ /group; combined data from the 40 and $100 \mathrm{mg} / \mathrm{kg} / \mathrm{d}$ experiments).

to tissue length (as described in Materials and Methods) was found to be greater in PG-PS-injected than in HSAinjected animals from these same experiments (Fig. 1C; $206.17 \pm 76.85$ vs. $60.91 \pm 7.44$, respectively; $P<0.02$; $n=9$ /group). Additionally, quantitative real-time PCR, performed on cecal tissue from the 40 and $100 \mathrm{mg} / \mathrm{kg} / \mathrm{d}$ experiments, demonstrated increased expression of procollagen types I and III mRNAs in PG-PS-injected as compared to HSA-injected animals (Fig 1D; procollagen type I: open bars, $8.35 \pm 1.91$ vs. $1.00 \pm 0.05$, respectively, $P<$ 0.001; procollagen type III: filled bars, $6.44 \pm 1.32$ vs. $1.00 \pm 0.03$, respectively, $P<0.001 ; n=9$ /group).

Representative trichrome-stained sections of cecum from HSA-injected (control; Fig. 2A,B) and PGPS-injected animals (vehicle-treated; Fig. 2C-F) are shown in Figure 2. Trichrome-stained sections from PG-PS-injected rats (vehicle-treated) demonstrated expansion of the submucosa and serosa with collagen (stained blue) that disrupts the muscularis externa (Fig. 2C,F). There were granulomas, some of which were necrotizing, in the submucosa (Fig. 2D). The mucosa has a mixed inflammatory cell infiltrate and occasional crypt abscesses (Fig. 2E and insert).

\section{Effects of Resveratrol in the PG-PS Model of CD Animal Weight}

There was no difference in the mean weight of resveratrol-treated and vehicle-treated PG-PS-injected rats in any of the three experiments or when animals from all experiments were considered collectively $(195.9 \pm 3.1 \mathrm{~g}$ vs. $195.6 \pm 2.9 \mathrm{~g}$, respectively; day 28 postinjection; $n=$ 16/group).

\section{Gross Abdominal Score}

Resveratrol had a dose-dependent effect on gross abdominal score in PG-PS-injected animals (Fig. 3). At 20 $\mathrm{mg} / \mathrm{kg} / \mathrm{d}$, resveratrol had no effect on gross abdominal score $(9.50 \pm 2.05$ vs. $9.33 \pm 1.63$, resveratrol- vs. vehicle-treated, respectively), and therefore no further analyses were performed on tissues from this experiment. There was a trend toward decreased gross abdominal score with 40 $\mathrm{mg} / \mathrm{kg} / \mathrm{d}$ resveratrol $(8.67 \pm 1.52$ vs. $10.00 \pm 2.41$, resveratrol- vs. vehicle-treated, respectively; $P=0.32$ ), and this trend was more prominent with the $100 \mathrm{mg} / \mathrm{kg} / \mathrm{d}$ dose $(13.80 \pm 2.29$ vs. $18.25 \pm 0.85$, resveratrol- vs. vehicletreated, respectively; $P=0.07)$. The mean percent decrease in gross abdominal score for the three resveratrol doses was $-1.8 \%, 13.3 \%$, and $24.4 \%$ for 20,40 , and $100 \mathrm{mg} / \mathrm{kg} /$ d, respectively (Fig. 3B), demonstrating a trend that was not statistically significant.

\section{Histologic Scoring of Inflammation and Fibrosis}

H\&E and trichrome-stained sections were examined in a blinded manner and scored for inflammation and fibrosis. There was no significant difference in inflammation score between resveratrol- and vehicle-treated PG-PSinjected animals with either 40 or $100 \mathrm{mg} / \mathrm{kg} / \mathrm{d}$ dosing $(10.8 \pm 1.4$ vs. $9.8 \pm 1.0$ and $10.6 \pm 1.8$ vs. $12.5 \pm 1.0$ $[P=0.21]$, respectively; Fig. 4A). A decrease in fibrosis score was seen with $100 \mathrm{mg} / \mathrm{kg} / \mathrm{d}$ dosing (6.4 \pm 1.1 vs. 9.8 \pm 1.0 , resveratrol- vs. vehicle-treated PG-PS-injected animals, respectively; $P=0.035$; Fig. 4B). Representative trichrome-stained sections of cecum from PG-PS-injected rats treated with resveratrol $(100 \mathrm{mg} / \mathrm{kg} / \mathrm{d})$ are shown in Figure $2 \mathrm{G}, \mathrm{H}$. Expansion of the submucosa and disruption of the muscularis externa by bands of collagen was less marked in the resveratrol-treated rats. 

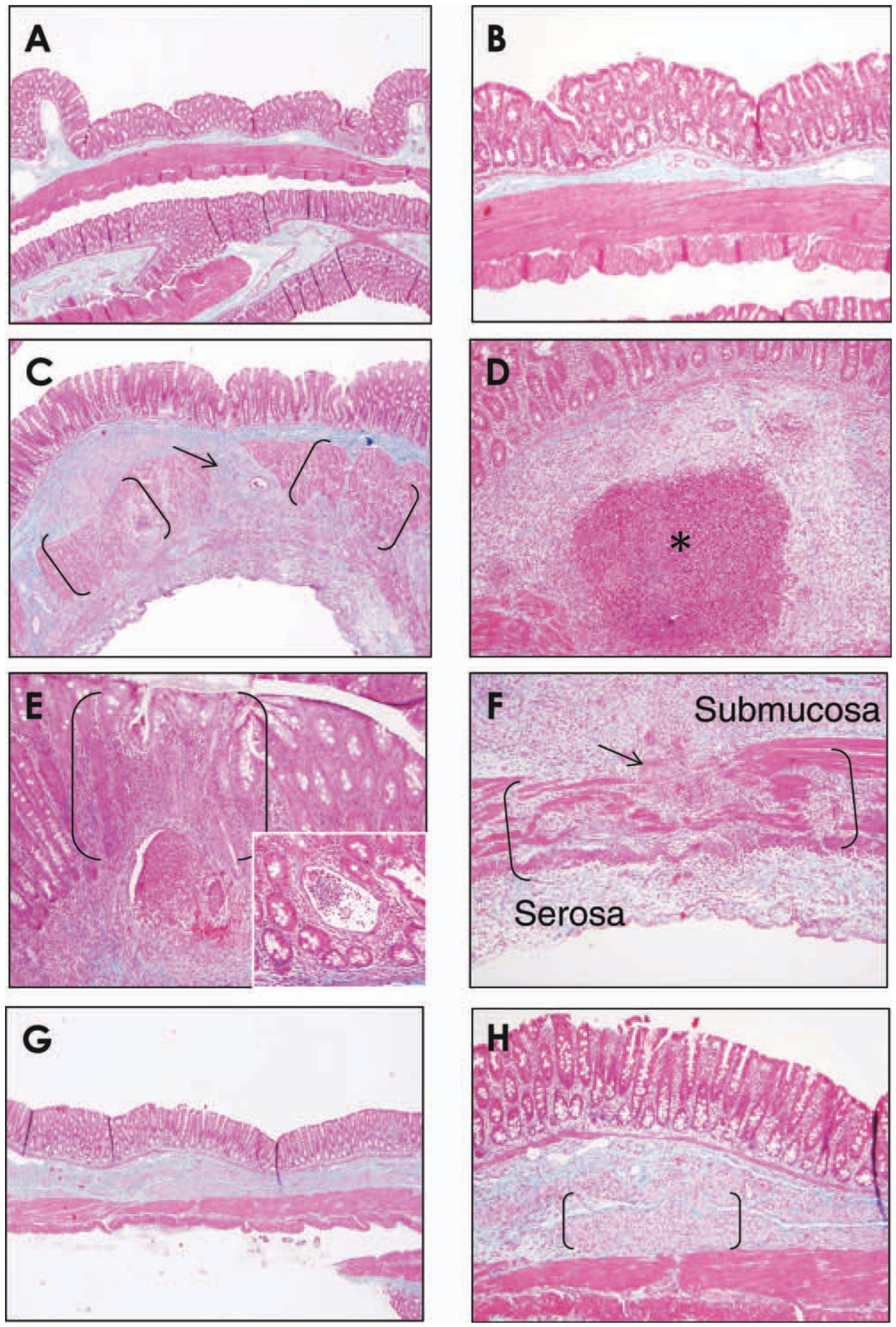

FIGURE 2. Microscopic appearance of representative trichrome-stained sections of cecum from HSA-injected control rats (A,B), PG-PSinjected rats (vehicle-treated; C-F), and PG-PS-injected rats treated with resveratrol 100/mg/kg/d (G,H). A,C,G are $\times 40$ original magnification photomicrographs of cecum from HSA-injected control rats, PG-PS-injected rats (vehicle-treated), and PG-PS-injected rats treated with resveratrol, respectively. B,D-F,H are $\times 100$ original magnification photomicrographs of cecum from HSA-injected control rats, PG-PS-injected rats (vehicle-treated), and PG-PS-injected rats treated with resveratrol, respectively. PG-PS-injected (vehicle-treated) rats have dense collagen (stained blue) expanding the submucosa and disrupting the muscularis externa (muscularis externa in brackets in C,F; disruption indicated by an arrow). Mucosal inflammation is shown in brackets in $E_{\text {; }}$ the insert is $\times 200$ original magnification of a crypt abscess. PG-PS-injected (vehicle-treated) rats have granulomas in the submucosa, many of which are necrotizing (asterisk in D). PG-PS-injected rats treated with resveratrol $100 / \mathrm{mg} / \mathrm{kg} / \mathrm{d}(\mathrm{G}, \mathrm{H})$ have less collagen in the submucosa and serosa, and the integrity of the muscularis externa is generally maintained compared with that of the PG-PS-injected (vehicle-treated) rats. The brackets in $\mathrm{H}$ indicate foamy histiocytes in the submucosa in the PG-PS-injected rats treated with resveratrol. [Color figure can be viewed in the online issue, which is available at wileyonlinelibrary.com.] 

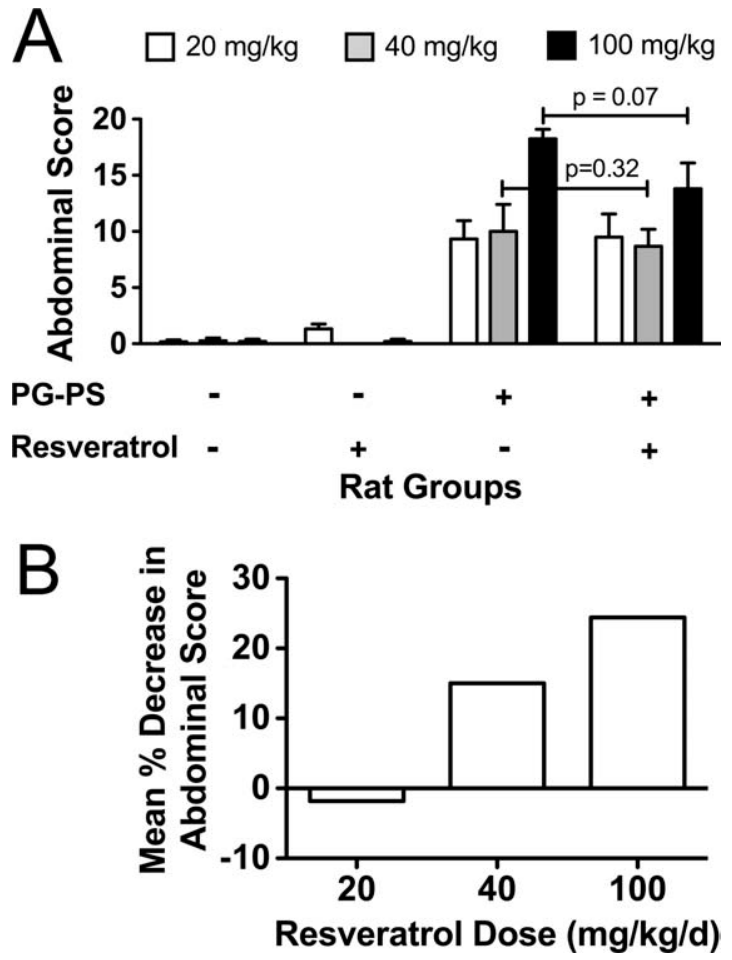

FIGURE 3. Effect of resveratrol on fibrosis as assessed by gross abdominal score. Resveratrol $(20,40$, or $100 \mathrm{mg} / \mathrm{kg} / \mathrm{d}$ ) or vehicle was given by daily gavage on days 1-27 postinjection to PG-PS- and HSA-injected rats. The animals were euthanized on day 28. (A) Gross abdominal scores were calculated (the sum of the scores [each on 0-4 scales] for adhesions, mesenteric thickening and contraction, cecal wall thickening, cecal nodules, and liver nodules; maximum possible score is 20). (Results shown are means; $n=4-$ 6/group; error bars reflect SEM.) (B) Results are shown as the mean percent decrease in gross abdominal score in PG-PS-injected animals that were treated with resveratrol relative to PG-PS-injected animals given vehicle.

\section{Colorimetric Analysis of Digital Images of Trichrome-stained Sections for Cecal Wall Collagen Content}

Trichrome-stained sections of cecum were analyzed for collagen content as described in Materials and Methods. At the $100 \mathrm{mg} / \mathrm{kg} / \mathrm{d}$ resveratrol dose, there was a trend toward decreased cecal collagen content with resveratrol treatment (Fig. 5; $142.19 \pm 20.63$ vs. $206.17 \pm 76.85$, resveratrol- vs. vehicle-treated PG-PS-injected animals, respectively; $P=0.16$ ).

\section{Proinflammatory Cytokines}

Using cDNA reverse transcribed from cecal RNA, quantitative real-time PCR was performed to measure expression of mRNAs of representative proinflammatory cytokines that are typically increased in inflamed tissue. At the $40 \mathrm{mg} / \mathrm{kg} / \mathrm{d}$ resveratrol dose (Fig. 6, gray bars) there was a trend toward decreased IL- $1 \beta$ and IL-6 mRNA expression in resveratrol-treated vs. vehicle-treated PG-PSinjected animals (IL-1 $\beta: 4.78 \pm 0.93$ vs. $8.13 \pm 3.00$,
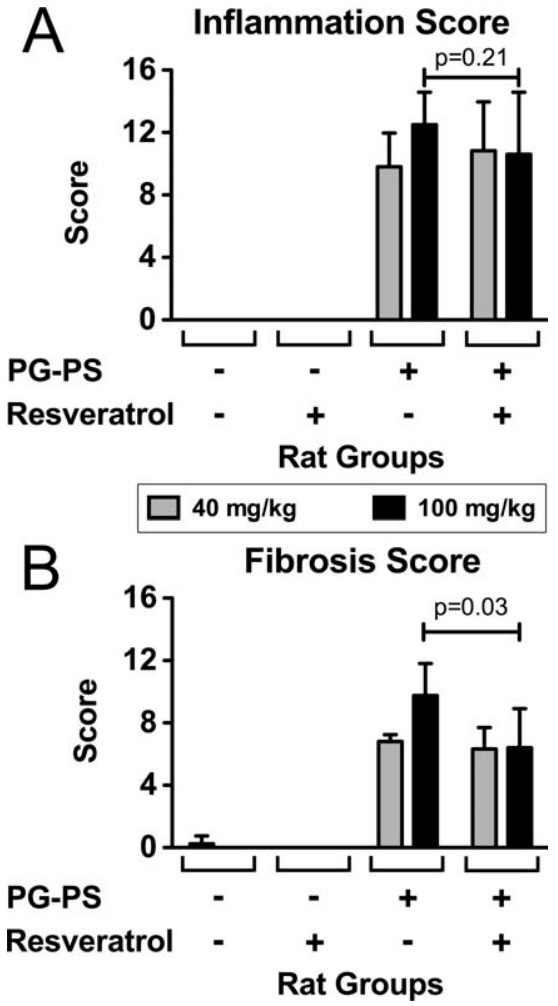

FIGURE 4. Effect of resveratrol on inflammation and fibrosis as assessed on histologic examination. H\&E and trichrome-stained sections were examined in blinded fashion and scored for inflammation (A) and for fibrosis (B) using a system outlined in Materials and Methods (Table 1). (Results shown are means; $n=4-6 /$ group; error bars reflect SEM.)

respectively, $P=0.14$; IL-6: $12.55 \pm 4.52$ vs. $22.02 \pm$ 8.05 , respectively, $P=0.16$ ), but no effect of resveratrol on TNF- $\alpha$ mRNA expression. At the $100 \mathrm{mg} / \mathrm{kg} / \mathrm{d}$

Trichrome Image Analysis

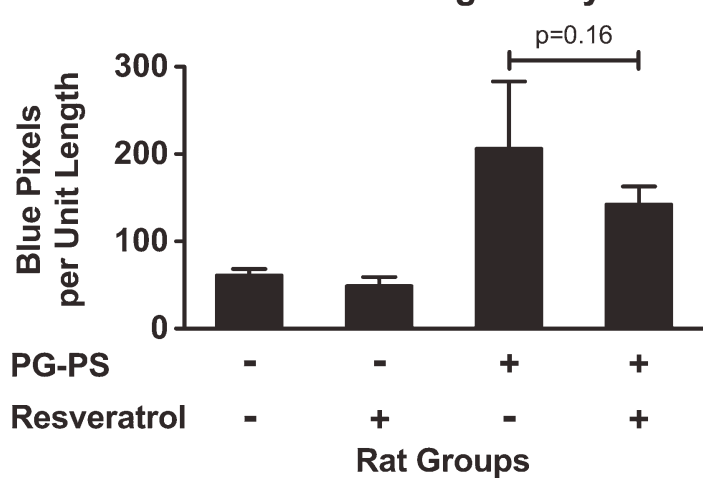

FIGURE 5. Effect of resveratrol on fibrosis using colorimetric analysis of digital images of trichrome-stained sections of cecum to measure collagen content. Digital images of trichrome-stained tissue sections were captured; color segmentation analysis was performed to count the number of blue pixels in each tissue section. The number of blue pixels was normalized to tissue section length to account for differences in the sizes of the pieces of tissue analyzed. (Results shown are means; $n=4-5$ /group; error bars reflect SEM.) 

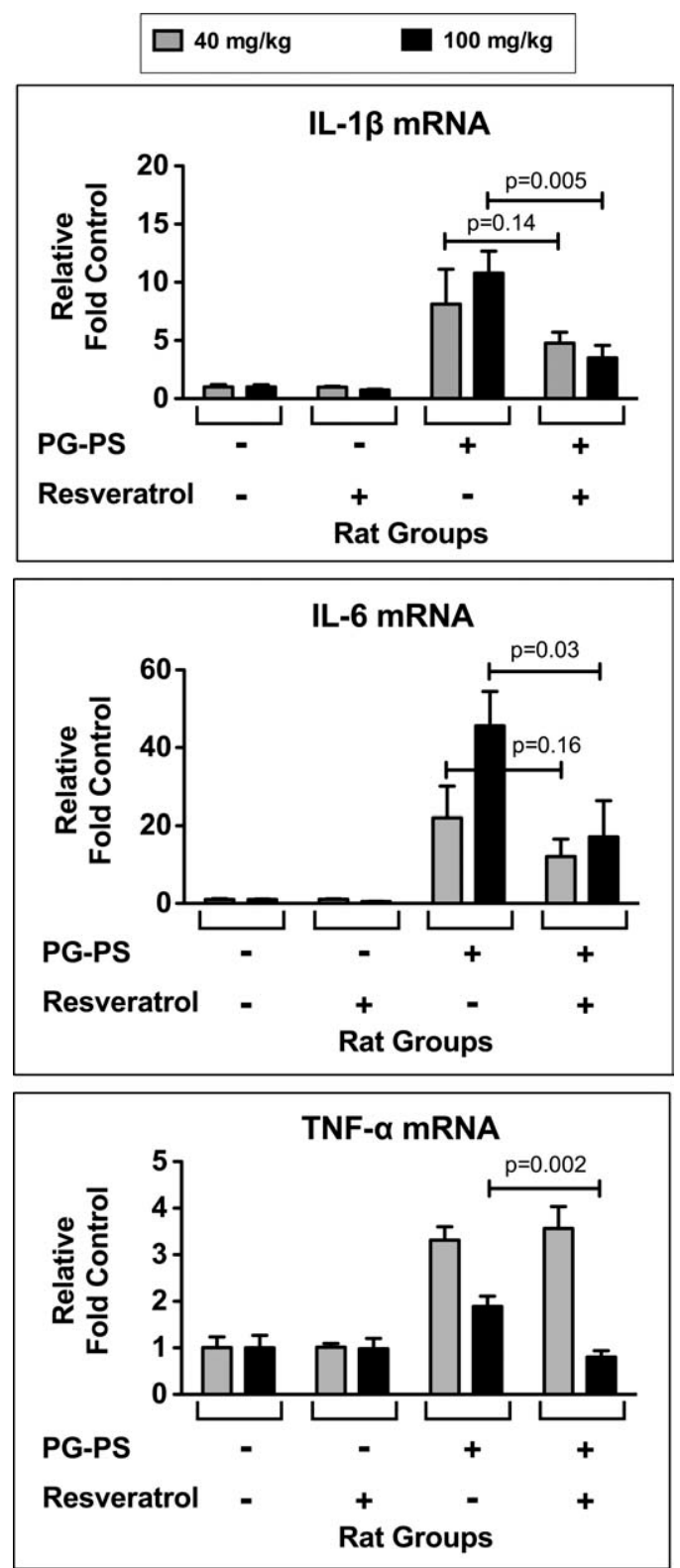

FIGURE 6. Effect of resveratrol on proinflammatory cytokines. Quantitative real-time PCR was used to measure IL-1 $\beta$, IL-6, and TNF- $\alpha$ mRNA expression in cecal tissue. The values for each specimen were normalized to the GAPDH mRNA level of that specimen. Results are shown relative to the mean for vehicle-treated HSAinjected animals. Statistical significance was achieved for all of these cytokines at the $100 \mathrm{mg} / \mathrm{kg} / \mathrm{d}$ dose. (Results shown are means; $n=4-6 /$ group; error bars reflect SEM.)

resveratrol dose (Fig. 6, black bars) the expression of the mRNAs of all three inflammatory cytokines was decreased (IL-1 $\beta$ : $3.50 \pm 1.08$ vs. $10.79 \pm 1.88, P=0.005$; IL-6: $17.11 \pm 9.22$ vs. $45.64 \pm 8.83, P=0.03$; TNF- $\alpha: 0.80 \pm$ 0.14 vs. $1.89 \pm 0.22, P=0.002$; resveratrol-treated vs. vehicle-treated PG-PS-injected animals, respectively).

\section{Profibrotic Factors}

There was a trend toward decreased IGF-I mRNA expression $(2.45 \pm 0.50$ vs. $3.10 \pm 0.70, P=0.23$; resveratrol-treated vs. vehicle-treated PG-PS-injected animals, respectively) with $40 \mathrm{mg} / \mathrm{kg} / \mathrm{d}$ resveratrol treatment (Fig. 7, gray bars). There was no effect on TGF- $\beta 1$, procollagen type I, or procollagen type III mRNA levels at the $40 \mathrm{mg} /$ $\mathrm{kg} / \mathrm{d}$ resveratrol dose. However, with the $100 \mathrm{mg} / \mathrm{kg} / \mathrm{d}$ dose (Fig. 7, black bars) TGF- $\beta 1$ mRNA expression was decreased (2.24 \pm 0.37 vs. $4.06 \pm 0.58$, resveratrol-treated vs. vehicle-treated PG-PS-injected animals, respectively; $P$ $=0.01$ ) and the other profibrotic factors that were measured showed a trend toward decreased expression (procollagen type I: $6.58 \pm 2.59$ vs. $12.47 \pm 2.96, P=0.09$; procollagen type III: $5.45 \pm 2.37$ vs. $9.03 \pm 2.08, P=0.16$; IGF-I: $4.01 \pm 1.11$ vs. $6.51 \pm 2.44, P=0.17$; resveratroltreated vs. vehicle-treated PG-PS-injected animals, respectively).

\section{DISCUSSION}

$\mathrm{CD}$ is a chronic relapsing inflammatory disease that involves primarily the distal small bowel and colon but can affect any part of the gastrointestinal tract. The characteristic transmural inflammatory state triggers smooth muscle hyperplasia and abnormal deposition of collagen and other extracellular matrix components resulting in fibrosis and luminal narrowing. ${ }^{32}$ Potent immunomodulators and biologic therapies are the mainstay of therapy for $\mathrm{CD}$. These therapies are highly effective in treating inflammation in most patients; however, the cost of the drugs and potential toxicities including life-threatening infections and cancer are concerning to both physicians and patients. Further, their effects on fibrosis and their ability to alter the natural history of the disease are not known. Indeed, there are no IBD therapies that have been specifically shown to decrease fibrosis. We investigated the ability of resveratrol, a naturally occurring phytochemical, to decrease inflammation and fibrosis in an animal model of CD. We found that resveratrol decreases inflammatory cytokines and profibrotic factors and decreases procollagen mRNAs, supporting a potential therapeutic benefit. The effects on inflammation and fibrosis in this model are modest, with some parameters demonstrating significance and others showing a trend, suggesting that resveratrol may be effective as an adjunct to current therapies rather than a primary therapeutic agent. Our data are consistent with a role for resveratrol as an effective, safe, economical, and "natural" adjunct to current therapies for CD.

Previous animal studies have demonstrated that resveratrol has antiinflammatory effects in several organ systems, ${ }^{10,11}$ including the gastrointestinal tract. ${ }^{12-18}$ The antioxidant and antiinflammatory actions of resveratrol 


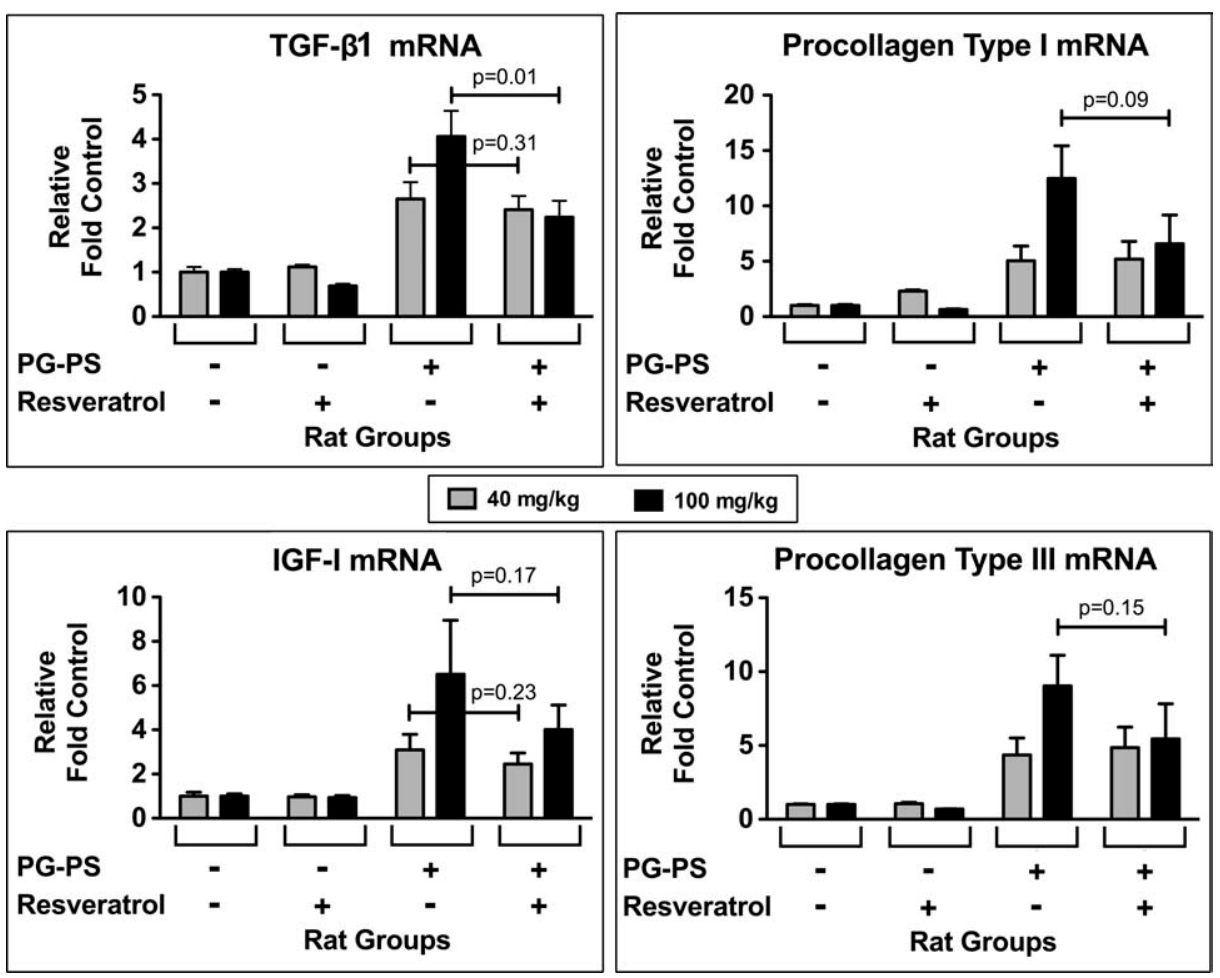

FIGURE 7. Effect of resveratrol on profibrotic factors. Quantitative real-time PCR was used to measure procollagen type I, procollagen type III, IGF-I, and TGF- $\beta 1$ mRNA expression in cecal tissue. The values for each specimen were normalized to the GAPDH mRNA level of that specimen. Results are shown relative to the mean for vehicle-treated HSA-injected animals. (Results shown are means; $n=4-6 / g r o u p ;$ error bars reflect SEM.)

occur via its inhibition of prostaglandin production, cyclooxygenase- 2 activity, and nuclear factor- $\kappa \mathrm{B}(\mathrm{NF}-\kappa \mathrm{B})$ activity. ${ }^{33}$ Resveratrol has also been shown to have antifibrotic effects in animal models of disease and injury involving the esophagus, liver, kidney, lung, heart, and vasculature, ${ }^{19-25,34}$ which is attributed in part to TGF- $\beta$ inhibition and reduction of oxidative stress ${ }^{19,20}$; the antifibrotic potential of resveratrol in animal models of IBD has not been previously assessed. The finding that resveratrol protected against stricture formation in an animal study of caustic esophageal burns ${ }^{18}$ suggests that resveratrol might protect against strictures elsewhere in the gastrointestinal tract, such as the intestinal strictures that frequently occur in CD.

Resveratrol decreases inflammation in the DSS (dextran sulfate sodium) $)^{12-16}$ and TNBS (2,4,6-trinitrobenzenesulfonic acid) ${ }^{17}$ rodent models of IBD. Both are better models of ulcerative colitis than $\mathrm{CD}$ because involvement in these models is restricted to the colon, and neither has significant fibrosis as performed using most experimental protocols. The PG-PS model more closely resembles CD with involvement in the terminal ileum and right colon, histologic features including granulomatous inflammation and prominent fibrosis, and extraintestinal manifestations such as arthritis that are commonly observed in CD patients. Further, the inflammation in this model is T-cell- mediated and has a similar inflammatory cytokine profile. ${ }^{27,28,35}$ Mechanistically, muramyl dipeptide, the substrate for the CD susceptibility gene product NOD2, ${ }^{36}$ is a moiety within the PG-PS macromolecule. Consistent with prior literature, the presence of inflammation and fibrosis, as well as involvement of the terminal ileum and colon, make this model relevant to $\mathrm{CD}$. Consistency and high penetrance of this model made it a good choice for drug testing. Based on the reports in the literature of resveratrol's antiinflammatory and antifibrotic properties, and the encouraging results of our in vitro experiments in which resveratrol-treated rat intestinal smooth muscle cells showed a decrease in collagen type I synthesis, ${ }^{26}$ we proceeded with the in vivo studies presented here.

A review of the literature ${ }^{6-9,12-25}$ indicated that a wide range of resveratrol doses had been used in in vivo rodent studies; physiologic and biochemical effects of resveratrol have been observed with doses between 0.2 and $100 \mathrm{mg} / \mathrm{kg} / \mathrm{d}$. With regard to toxicity, it has been shown that a resveratrol dose of $300 \mathrm{mg} / \mathrm{kg} / \mathrm{d}$ for 28 days in rats had no adverse effects. ${ }^{37,38}$ A dosage range of $20-100 \mathrm{mg} /$ $\mathrm{kg} / \mathrm{d}$ was chosen for our studies in the PG-PS model. There was a dose-dependent decrease in gross abdominal score (Fig. 3). At the $100 \mathrm{mg} / \mathrm{kg} / \mathrm{d}$ dose there was a significant reduction in proinflammatory cytokine mRNAs (Fig. 6). 
This observation is in agreement with work by others using other models of IBD in rats: Larrosa et $\mathrm{al}^{12}$ in the DSS model and Martín et $\mathrm{al}^{17}$ in the TNBS model found that resveratrol reduced the intestinal inflammatory response to those inducing agents. Similar antiinflammatory effects of resveratrol have been reported when the DSS model is carried out in mice. ${ }^{13-16}$ Singh et $\mathrm{al}^{15}$ found that $100 \mathrm{mg} / \mathrm{kg}$ resveratrol administered every other day by gavage, without a pretreatment period, was required for protection of mice from DSS colitis. Our finding of significant reductions in the levels of inflammatory cytokine mRNAs (Fig. 6) but only a trend toward a decrease in the histologic inflammation score (Fig. 4A) may reflect factors including differences in the sensitivities of the assessment tools or, perhaps, an early decrease in the level of activation of the inflammatory cells that would be reflected in decreased tissue inflammation at a later timepoint.

Resveratrol at a dose of $100 \mathrm{mg} / \mathrm{kg} / \mathrm{d}$ decreased tissue fibrosis in the PG-PS model. Reduction in fibrosis was seen on histologic examination (Fig. 4B) and was supported by reduced TGF- $\beta 1$ mRNA levels (Fig. 7) and by trends toward lower gross abdominal score (Fig. 3), lower cecal wall collagen content on colorimetric analysis of digital images of trichrome-stained sections (Fig. 5), and decreased procollagen type I, procollagen type III, and IGF-I mRNA levels (Fig. 7). The finding of decreased fibrosis with resveratrol treatment is consistent with previous reports of antifibrotic effects of resveratrol in a variety of in vivo models of disease. ${ }^{18-25,34}$ The decrease in TGF- $\beta 1$ mRNA in our study is in agreement with previous studies that have demonstrated a decrease in the level of this profibrotic factor with resveratrol treatment, and is consistent with the proposed mechanism of resveratrol's antifibrotic action. ${ }^{19,20,21}$ TGF- $\beta 1$ is a potent profibrotic factor that plays a role in the pathogenesis of CD strictures. ${ }^{32}$

There was a trend toward a decrease in IGF-I mRNA with resveratrol treatment in our study. IGF-I has potent profibrotic effects and data suggest that it plays a role in the pathogenesis of strictures in CD. ${ }^{39,40}$ The IGF-I system also plays a role in the development of cancer, particularly colon cancer. ${ }^{41,42}$ While we did not directly assess chemoprevention in our study, resveratrol's chemopreventive effects have been reported in a number of in vitro and in vivo models. ${ }^{2,3,6-9,13,33}$ Relevant to IBD, these reports include chemopreventive effects in colon. ${ }^{7,8,13} \mathrm{~A}$ recent study in humans, comparing pre- and postintervention tissue, demonstrated decreased tumor cell proliferation in tissue from colorectal cancer patients given resveratrol for the 8 days prior to surgical resection of their tumor. ${ }^{43}$ It was also recently shown that serum IGF-I levels decreased in volunteers taking resveratrol. ${ }^{44}$ The effect of resveratrol on IGF-I is especially relevant to CD since IGF-I has been implicated in intestinal fibrosis in this disease $\mathrm{s}^{39,40}$ and since patients with colonic CD have an increased risk of colon cancer. ${ }^{45}$

Relevant to treatment of $\mathrm{CD}$, enterohepatic circulation of resveratrol has been demonstrated experimentally, ${ }^{46}$ increasing exposure of the gut to resveratrol. However, human and rat pharmacokinetic studies have shown that resveratrol is rapidly and extensively metabolized and has low bioavailability. ${ }^{44,47,48}$ In humans, achievable blood levels of unconjugated resveratrol are in the range of 0.02-0.5 $\mu \mathrm{M}$ when doses of $0.6-5.0 \mathrm{~g} / \mathrm{d}$ are given. ${ }^{5,44,48,49}$ Circulating levels of its major metabolites (resveratrol-3-O-sulfate, resveratrol-4'-O-glucuronide, and resveratrol-3-O-glucuronide) have been found to be much higher than levels of the parent compound. ${ }^{44}$ Despite emerging evidence that at least some resveratrol metabolites may be pharmacologically active, ${ }^{4,44,50,51}$ recent studies suggest that resveratrol doses of $0.5-2.5 \mathrm{~g} / \mathrm{d}$ may be required for chemopreventive efficacy in human colon. ${ }^{43,44}$ Doses of 2.5 and $5.0 \mathrm{~g}$ once daily were safe, but caused mild to moderate gastrointestinal symptoms. ${ }^{44}$ While administration as divided doses might decrease the occurrence of side effects, other approaches may be useful to allow lower resveratrol doses to be pharmacologically effective. It has been reported that flavonoids such as quercitin inhibit the conjugation of resveratrol and thereby increase resveratrol bioavailability ${ }^{52}$ suggesting that dietary manipulations could perhaps allow therapeutic blood and tissue levels of resveratrol to be more readily achieved. Increasing the aqueous solubility of resveratrol was found to increase the rate of absorption but not the bioavailability of orally administered resveratrol. ${ }^{53}$ More recently, the development of resveratrol proprodrugs was found to improve the efficacy of resveratrol in preventing the development of DSS-induced colitis in mice. $^{54}$

Although it has been reported that resveratrol modulates the expression of some cytochrome P450 and other drug metabolizing enzymes, ${ }^{4,5}$ and could therefore affect pharmacokinetics of certain drugs, resveratrol is generally safe with minimal side effects. ${ }^{44,48}$ The human equivalent dose $^{55}$ of a $100 \mathrm{mg} / \mathrm{kg} / \mathrm{d}$ dose in rats is $16 \mathrm{mg} / \mathrm{kg} / \mathrm{d}$, or 1.12 $\mathrm{g} / \mathrm{d}$ for a $70-\mathrm{kg}$ individual. Thus, based on available literature and our rodent study, it is not unreasonable to conclude that concentrations of resveratrol needed to decrease fibrosis in CD can be attained in the blood or target tissue in humans. However, definitive studies will need to be performed in $\mathrm{CD}$ patients.

In conclusion, we found that resveratrol decreased cecal wall fibrosis as assessed histologically and this was associated with a decrease in the profibrotic cytokine TGF$\beta 1$ as well as a number of inflammatory cytokines (IL-1 $\beta$, IL-6, and TNF- $\alpha$ ) in the PG-PS rat model of CD. Promising trends toward decreased expression of other profibrotic factors (IGF-I, procollagen type I, and procollagen type III 
mRNAs), decreased gross abdominal score, decreased cecal wall collagen content, and decreased histologic inflammation score were also demonstrated. These results, together with resveratrol's reported chemopreventive effects, suggest that resveratrol may be a valuable adjunct to our standard immunomodulator therapies for CD. Translational studies investigating the effect of this compound in $\mathrm{CD}$ patients would be the next step.

\section{REFERENCES}

1. Goswami SK, Das DK. Resveratrol and chemoprevention. Cancer Lett. 2009;284:1-6.

2. Jang M, Cai L, Udeani GO, et al. Cancer chemopreventive activity of resveratrol, a natural product derived from grapes. Science. 1997;275: $218-220$

3. Sgambato A, Ardito R, Faraglia B, et al. Resveratrol, a natural phenolic compound, inhibits cell proliferation and prevents oxidative DNA damage. Mutat Res. 2001;496:171-180.

4. Baur JA, Sinclair DA. Therapeutic potential of resveratrol: the in vivo evidence. Nat Rev Drug Discov. 2006;5:493-506.

5. de la Lastra CA, Villegas I. Resveratrol as an anti-inflammatory and anti-aging agent: Mechanisms and clinical implications. Mol Nutr Food Res. 2005;49:405-430.

6. Li ZG, Hong T, Shimada Y, et al. Suppression of N-nitrosomethylbenzylamine (NMBA)-induced esophageal tumorigenesis in F344 rats by resveratrol. Carcinogenesis. 2002;23:1531-1536.

7. Sengottuvelan M, Viswanathan P, Nalini N. Chemopreventive effect of trans-resveratrol - a phytoalexin - against colonic aberrant crypt foci and cell proliferation in 1,2-dimehtylhydrazine induced colon carcinogenesis. Carcinogenesis. 2006;27:1038-1046.

8. Tessitore L, Davit A, Sarotto I, et al. Resveratrol depresses the growth of colorectal aberrant crypt foci by affecting bax and p21CIP expression. Carcinogenesis. 2000;21:1619-1622.

9. Banerejee S, Bueso-Ramos C, Aggarwal BB. Suppression of 7,12dimethylbenz(a)anthracene-induced mammary carcinogenesis in rats by resveratrol: role of nuclear factor- $\mathrm{\kappa B}$, cyclooxygenase 2 , and matrix metalloprotease 9. Cancer Res. 2002;62:4945-4954.

10. Wood LG, Wark PAB, Garg ML. Antioxidant and anti-inflammatory effects of resveratrol in airway disease. Antioxid Redox Signal. 2010; 13:1535-1548.

11. Losso JN, Truax RE, Richard G. trans-Resveratrol inhibits hyperglycemia-induced inflammation and connexin downregulation in retinal pigment epithelial cells. J Agric Food Chem. 2010;58:8246-8252.

12. Larrosa M, Yañéz-Gascón MJ, Selma MV, et al. Effect of a low dose of dietary resveratrol on colon microbiota, inflammation and tissue damage in a DSS-induced colitis rat model. J Agric Food Chem. 2009;57:2211-2220.

13. Cui X, Jin Y, Hofseth AB, et al. Resveratrol suppresses colitis and colon cancer associated with colitis. Cancer Prev Res. 2010;3:549-559.

14. Youn J, Lee J-S, Na H-K, et al. Resveratrol and piceatannol inhibit iNOS expression and NF- $\mathrm{kB}$ activation in dextran sulfate sodiuminduced mouse colitis. Nutr Cancer. 2009;61:847-854.

15. Singh UP, Singh NP, Singh B, et al. Resveratrol (trans-3,5,4'-trihydroxystilbene) induces silent mating type information regulation- 1 and down-regulates nuclear transcription factor- $\mathrm{\kappa B}$ activation to abrogate dextran sulfate sodium-induced colitis. J Pharmacol Exp Ther. 2010; 332:829-839.

16. Sánchez-Fidalgo S, Cárdeno A, Villegas I, et al. Dietary supplementation of resveratrol attenuates chronic colonic inflammation in mice. Eur J Pharmacol. 2010;633:78-84.

17. Martín AR, Villegas I, Sánchez-Hidalgo M, et al. The effects of resveratrol, a phytoalexin derived from red wines, on chronic inflammation induced in an experimentally induced colitis model. Br J Pharmacol. 2006;147:873-885

18. Uguralp S, Irsi C, Aksoy T, et al. Resveratrol attenuates inflammation and stricture formation in experimental caustic esophageal burns. Pediatr Surg Int. 2008;24:425-430.
19. Chavez E, Reyes-Gordillo K, Segovia J, et al. Resveratrol prevents fibrosis, NF- $\mathrm{kB}$ activation and TGF- $\beta$ increases induced by chronic CCL4 treatment in rats. J Appl Toxicol. 2008;28:35-43.

20. Lee E-S, Shin M-O, Yoon S, et al. Resveratrol inhibits dimethylnitrosamine-induced hepatic fibrosis in rats. Arch Pharm Res. 2010;33: 925-932.

21. Hong S-W, Jung KH, Zheng H-M, et al. The protective effect of resveratrol on dimethylnitrosamine-induced liver fibrosis in rats. Arch Pharm Res. 2010;33:601-609.

22. Li J, Qu X, Ricardo SD, et al. Resveratrol inhibits renal fibrosis in the obstructed kidney. Am J Pathol. 2010;177:1065-1071.

23. Sutra T, Oiry C, Azay-Milhau J, et al. Preventive effects of nutritional doses of polyphenolic molecules on cardiac fibrosis associated with metabolic syndrome: involvement of osteopontin and oxidative stress. J Agric Food Chem. 2008;56:11683-11687.

24. Jing Y-H, Chen K-H, Yang S-H, et al. Resveratrol ameliorates vasculopathy in STZ-induced diabetic rats: role of AGE-RAGE signalling. Diabetes Metab Res Rev. 2010;26:212-222.

25. Zou J, Huang Y, Cao K, et al. Effect of resveratrol on intimal hyperplasia after endothelial denudation in an experimental rabbit model. Life Sci. 2000;68:153-163.

26. Garcia P, Schmiedlin-Ren P, Mathias JS, et al. Resveratrol causes cell cycle arrest, decreased collagen synthesis, and apoptosis in rat intestinal smooth muscle cells. Am J Physiol Gastrointest Liver Physiol. (in press).

27. Sartor RB, Cromartie WJ, Powell DW, et al. Granulomatous enterocolitis induced in rats by purified bacterial cell wall fragments. Gastroenterology. 1985;89:587-596.

28. Zimmermann EM, Sartor RB, McCall RD, et al. Insulin-like growth factor I and interleukin $1 \beta$ messenger RNA in a rat model of granulomatous enterocolitis and hepatitis. Gastroenterology. 1993;105: 399-409.

29. Adler J, Swanson SD, Schmiedlin-Ren P, et al. Magnetization transfer helps detect intestinal fibrosis in an animal model of Crohn disease. Radiology. 2011;259:127-135.

30. International Commission on Illumination. Proceedings of the CIE Expert Symposium '97 on Colour Standards for Image Technology. Vienna, Austria: Commission Internationale de l'éclairage, CIE Central Bureau, 1998.

31. Schwartz MW, Cowan WB, Beatty JC. An experimental comparison of RGB, YIQ, LAB, HSV, and opponent color models. ACM Trans Graph. 1987;6:123-158.

32. Burke JP, Muslow JJ, O'Keane C, et al. Fibrogenesis in Crohn's disease. Am J Gastroenterol. 2007;102:439-448.

33. Shankar S, Gyanendra G, Srivastava RK. Chemoprevention by resveratrol: molecular mechanisms and therapeutic potential. Frontiers Biosci. 2007;12:4839-4854.

34. Kolgazi M, Şener G, Çetinel Ş, et al. Resveratrol reduces renal and lung injury caused by sepsis in rats. J Surg Res. 2006;134: 315-321.

35. Isaacs KL, Sartor RB, Haskill S. Cytokine messenger RNA profiles in inflammatory bowel disease mucosa detected by polymerase chain reaction amplification. Gastroenterology. 1992;103:1587-1595.

36. Inohara N, Ogura Y, Fontalba A, et al. Host recognition of bacterial muramyl dipeptide mediated through NOD2. Implications for Crohn's disease. J Biol Chem. 2003;278:5509-5512.

37. Crowell JA, Korytko PJ, Morrissey RL, et al. Resveratrol-associated renal toxicity. Toxicol Sci. 2004;82:614-619.

38. Hebbar V, Shen G, Hu R, et al. Toxicogenomics of resveratrol in rat liver. Life Sci. 2005;76:2299-2314.

39. Zimmermann EM, Li L, Hou YT, et al. Insulin-like growth factor I and insulin-like growth factor binding protein 5 in Crohn's disease. Am J Physiol Gastrointest Liver Physiol. 2001;280:G1022-G1029.

40. Pucilowska JB, McNaughton KK, Mohapatra NK, et al. IGF-I and procollagen a1(I) are co-expressed in a subset of mesenchymal cells in active Crohn's disease. Am J Physiol Gastrointest Liver Physiol. 2000;279:G1307-G1322.

41. Yu H, Rohan T. Role of the insulin-like growth factor family in cancer development and progression. J Natl Cancer Inst. 2000;92: $1472-1489$. 
42. Sandhu MS, Dunger DB, Giovannucci EL. Insulin, insulin-like growth factor-I (IGF-I), IGF binding proteins, their biologic interactions, and colorectal cancer. J Natl Cancer Inst. 2002;94:972-980.

43. Patel KR, Brown VA, Jones DJL, et al. Clinical pharmacology of resveratrol and its metabolites in colorectal cancer patients. Cancer Res. 2010;70:7392-7399.

44. Brown VA, Patel KR, Viskaduraki M, et al. Repeat dose study of the cancer chemopreventive agent resveratrol in healthy volunteers: safety, pharmacokinetics, and effect on the insulin-like growth factor axis. Cancer Res. 2010;70:9003-9011.

45. Xie J, Itzkowitz SH. Cancer in inflammatory bowel disease. World J Gastroenterol. 2008; 14:378-389.

46. Marier J-F, Vachon P, Gritsas A, et al. Metabolism and disposition of resveratrol in rats: extent of absorption, glucuronidation, and enterohepatic recirculation evidenced by a linked-rat model. J Pharmacol Exp Ther. 2002;302:369-373.

47. Yu C, Shin YG, Chow A, et al. Human, rat, and mouse metabolism of resveratrol. Pharm Res. 2002;19:1907-1914.

48. Boocock DJ, Faust GES, Patel KR, et al. Phase I dose escalation pharmacokinetic study in healthy volunteers of resveratrol, a potential can- cer chemopreventive agent. Cancer Epidemiol Biomarkers Prev. 2007; 16:1246-1252.

49. Almeida L, Vaz-da-Silva M, Falcão A, et al. Pharmacokinetic and safety profile of trans-resveratrol in a rising multiple-dose study in healthy volunteers. Mol Nutr Food Res. 2009;53:S7-S15.

50. Calamini B, Ratia K, Malkowski MG, et al. Pleiotropic mechanisms facilitated by resveratrol and its metabolites. Biochem J. 2010;429:273-282.

51. Hoshino J, Park E-J, Kondratyuk TP, et al. Selective synthesis and biological evaluation of sulfate-conjugated resveratrol metabolites. J Med Chem. 2010;53:5033-5043.

52. De Santi C, Pietrabissa A, Mosca F, et al. Glucuronidation of resveratrol, a natural product present in grape and wine, in the human liver. Xenobiotica. 2000;30:1047-1054.

53. Das S, Lin H-S, Ho PC, et al. The impact of aqueous solubility and dose on the pharmacokinetic profiles of resveratrol. Pharm Res. 2008;25:2593-2600.

54. Larrosa M, Tomé-Carneiro J, Yáñez-Gascón MJ, et al. Preventive oral treatment with resveratrol pro-prodrugs drastically reduce colon inflammation in rodents. J Med Chem. 2010;53:7365-7376.

55. Reagan-Shaw S, Nihal M, Ahmad N. Dose translation from animal to human studies revisited. FASEB J. 2007;22:659-661. 